$$
\text { SAND- } 96-0236 C \quad \text { CONF.960611-.1 }
$$

\title{
A COMPACT TRANSPORTABLE JOSEPHSON VOLTAGE STANDARD*
}

\author{
Clark A. Hamilton and Charles J. Burroughs \\ National Institute of Standards and Technology \\ Boulder, CO 80303-3328
}

\author{
Stuart L. Kupferman \\ Sandia National Laboratories \\ Albuquerque, NM 87185-0665 \\ Gerald Naujoks \\ Keithley Instruments Inc. \\ Solon, OH 44139-1891 \\ Andrew Vickery \\ Millitech Corporation \\ South Deerfield, MA 01373
}

\begin{abstract}
$\underline{\text { Abstract }}$
The development of a compact, portable $10 \mathrm{~V}$ Josephson calibration system is described. Its accuracy is the same as typical laboratory systems and its weight and volume are reduced by more than a factor of three. The new system will replace travelling voltage standards used within several NASA and DOE standards laboratories.
\end{abstract}

\section{Introduction}

A new Josephson Voltage Standard (JVS), with a weight and volume of only $21 \mathrm{~kg}$ and $0.03 \mathrm{~m}^{3}$, has been developed. The system is highly automated and is designed to be operated by technicians without higher level support. In contrast to typical laboratory systems that are semipermanent installations, the new system is easily shipped in two custom shipping containers and can be set up in less than an hour. The first prototype will be cooled by liquid helium with a 100 liter Dewar that will be sufficient to operate the Josephson array for six to eight weeks. The final version is designed to be cooled with a compact portable 4-K refrigeration system currently under development.

\section{System Design}

As shown in Fig.1, the system has three components: (1) a notebook computer, (2) an electronics package in a single 13-cm high rack-width box, and (3) a cryoprobe adaptable to fit in a wide variety of Dewars. In addition, two solid-state voltage standards, used as check standards, travel with the system. Figure 2 is a functional diagram of the system. A notebook computer controls the system through the parallel port. A commercially available IEEE interface splits the parallel port into both a printer port and an IEEE port. The IEEE port controls a low thermal scanner, external instruments

*Contribution of the US Government, not subject to copyright. This work was supported by the National Aeronautics and Space Administration (NASA) under Funding Document CC72715B, by the United States Department of Energy (DOE) under Contract DE-AC04-94AL8500, and by the U.S. Army under CCG project 346. 


\section{DISCLAMER}

Portions of this document may be illegible in electronic image products. Images are produced from the best available original document. 
such as digital voltmeters under test and an internal DVM module that was especially developed for the compact JVS. The DVM module includes two fixed range, 7-digit meters $(10 \mathrm{~V}$ and $10 \mathrm{mV})$ and a serial output data port. The $10 \mathrm{~V}$ meter uses a 7-channel relay multiplexer to monitor and record the system environment (temperature, humidity, and atmospheric pressure) as well as to make diagnostic measurements on the Josephson array and its bias system. The internal temperature of the electronics package is monitored and stabilized by a variable speed fan. The $10 \mathrm{mV}$ meter has a noise level of $18 \mathrm{nV}$ in a $1 \mathrm{~Hz}$ bandwidth and is used as the null meter for measurements of dc reference standards. The serial data port provides the digital codes to control the multiplexer, system control relays, a 16-bit digital to analog converter (DAC), and the microwave attenuator servo. The five system control relays provide the following functions: (1) Bias current to the Josephson array is selected to be either a manual, variable-impedance dc offset and sweep generator, or the output of a 16-bit DAC. (2) A $10 \mathrm{k} \Omega$ resistor can be substituted in place of the array in order to provide self-calibration of the bias current and to disconnect the bias circuit from the array during null measurements. (3) An open circuit can be substituted for the array to provide self calibration of the bias voltage. (4) A $120 \mathrm{~V}$ ac outlet can be switched on and off so that dc standards under test may be automatically put on battery power during measurements. (5) The $77 \mathrm{GHz}$ Gunn oscillator can be switched on and off.

\section{Cryoprobe and Microwave Source}

The cryoprobe for the new system, shown at the right side of Fig. 2, differs from previous designs [1 ] in two respects: First, the $77 \mathrm{GHz}$ source and RFI filters in the array bias and potential leads are all enclosed within several different compartments of a single rf-tight box at the top of the cryoprobe. Since this source is fixed frequency and the attenuator is servo controlled, there are no user adjustments on the microwave system. Second, microwave power is delivered to the Josephson array through a rigid dielectric waveguide constructed from a $25 \mathrm{~mm}$ dia. Teflon tube with a rectangular $2 \times 4 \mathrm{~mm}$ solid Teflon core as shown in Fig. 4. The core is centered in the tube by eight Teflon membrane spacers. The ends of the core are tapered at $15^{\circ}$ and pushed into WR-12 horns attached to each end of the outer Teflon tube. Typical attenuation of this $1.25 \mathrm{~m}$ long waveguide is $2.2 \mathrm{~dB}$ at 75 $\mathrm{GHz}$. The six bias and potential leads are enclosed in two 1-mm diameter stainless-steel tubes enclosed within the waveguide tube. This dielectric waveguide design is more economical than commercial flexible dielectric waveguides and it is easily disassembled for modification or repair. Since condensation on the core causes a substantial increase in attenuation, care must be taken to keep the waveguide sealed and slightly over pressurized with dry helium gas from the liquid helium Dewar.

The microwave source is a fixed frequency, $77 \mathrm{GHz}$, phase locked oscillator (PLO). A block diagram of the PLO is shown in Fig. 3a. Its $10 \mathrm{MHz}$ reference frequency comes from either a high stability internal quartz crystal oscillator or (preferably) a $10 \mathrm{MHz}$ frequency standard derived from a rubidium clock or a GPS receiver. The local oscillator at $7.690 \mathrm{GHz}$ is phase locked to the $10 \mathrm{MHz}$ reference frequency. The $77 \mathrm{GHz}$ Gunn oscillator output is mixed with the tenth harmonic of the local oscillator to generate an IF at $100 \mathrm{MHz}$. The phase difference between the IF and the tenth harmonic of the reference frequency is the error signal that is used to bias tune the Gunn oscillator and establish the phase lock. The wide bandwidth of the phase lock loop in this system results in a phase noise of -55 $\mathrm{dBc}$ at a $2 \mathrm{KHz}$ offset from the carrier. This is three orders of magnitude improvement over the phase noise that is achieved with the microwave counters used to stabilize the frequency in typical Josephson voltage standard systems. Figure $3 \mathrm{~b}$ compares the spectral line width of our system and a typical microwave counter stabilized source. The $77 \mathrm{GHz}$ power is controlled by a servo driven vane attenuator. 


\section{$\underline{\text { Software }}$}

Software for the system has the same calibration and data display algorithms used in the program NISTVolt version 5.0 [2] and is based on the circuit in Fig. 5. Here, $V_{\mathrm{a}}=n f / K_{\mathrm{j}}$ is the Josephson array voltage, $V_{z}$ is the best estimate of the unknown reference voltage, $V_{0}$ is a combination of thermal offset voltages and any offset voltage in the null meter, $m t$ represents a linear drift component of the offset voltage, $P=+-1$ is the polarity of the reversing switch, $V_{\mathrm{e}}$ is an initial direct measurement of $V_{\mathrm{z}}$ by the system $10-\mathrm{V}$ meter, $V_{\text {null }}$ is the null voltage measured by the system $10-\mathrm{mV}$ meter and $V_{\text {noise }}$ represents noise in the unknown, the null meter, and any other random noise contributions. The circuit equation can be written:

$$
V_{\mathrm{a}}-V_{\text {null }}=n f / K_{\mathrm{j}}-\mathrm{V}_{\text {null }}=P V_{\mathrm{z}}-V_{\mathrm{o}}-m T-V_{\text {noise }}
$$

Based on measurements of $V_{\mathrm{e}}$ and $V_{\text {null }}$ a set of 12 values of $V_{\mathrm{i}}$ and $T_{\mathrm{i}}$ is accumulated:

$$
\begin{aligned}
& V_{\mathrm{i}}=n f / K_{\mathrm{j}}-V_{\text {null }} \quad \mathrm{i}=1 \text { to } 12 \\
& T_{\mathrm{i}}=\text { the elapsed time at which } V_{\text {null }} \text { is measured. } \\
& P_{\mathrm{i}}=+1=\text { initial polarity of reversing switch. }
\end{aligned}
$$

The integer $n$ is determined from $V_{\text {null }}$ and the initial measurement $V_{\mathrm{e}}$ :

$$
n=\operatorname{Round}\left\{\left(K_{\mathrm{j}} / f\right)\left(V_{\mathrm{e}}+V_{\text {null }}\right)\right\},
$$

and the "Round" function means rounded to the nearest integer. For each of the 12 data points, 3 successive values of $V_{\text {nul }}$ are examined for consistency within $2 \mu \mathrm{V}$ before the data are accepted. This eliminates data that may be corrupted by the transient that occurs when there is a spontaneous transition between quantum voltage steps. Since $V_{\mathrm{a}}$ and $V_{\text {null }}$ change by equal amounts during a step transition, $V_{\mathrm{i}}$ remains constant thus making the data collection process relatively immune to step transitions. Data are collected efficiently even for a Josephson array chip that may be making as many as 5 transitions per minute. The scatter in the data that results from noise in the unknown and in the null meter can generally be modeled by a Gaussian process with one sigma on the order of 20-100 $\mathrm{nV}$. There are however occasional noise spikes that do not fit this process and generate glitches in the $V_{\mathrm{i}}$ data that may lie 1-10 uV away from the well behaved data. For this reason, the set of 12 points is reduced to 10 points by eliminating the two points furthest from the mean.

After the collection of the first data set, the polarity of the unknown is reversed $\left(P_{\mathrm{i}}=-1\right)$ and a second set of data is accumulated for $\mathrm{i}=11$ to 20 . Two more reversals generate data for $P_{\mathrm{i}}=+1, \mathrm{i}=21$ to 30 , and $P_{\mathrm{i}}=-1, \mathrm{i}=31-40$. The change from a +--+ reversal sequence to a +-+- sequence in this version of NISTVolt reduces some potential uncertainty resulting from an asymmetric influence of dielectric absorption and noise averaging.

The 40 data points are used in a 3 parameter least squares fit to obtain best estimates of $V_{z}, V_{0}$, and $m$. The matrix equation for the best estimate values is: 


$$
\left[\begin{array}{ccc}
N & 0 & \sum P_{i} T_{i} \\
0 & N & \sum T_{i} \\
\sum P_{i} T_{i} & \sum T_{i} & \sum T_{i}^{2}
\end{array}\right]\left[\begin{array}{c}
V_{z} \\
V_{0} \\
m
\end{array}\right]=\left[\begin{array}{c}
\sum P_{i} V_{i} \\
\sum V_{i} \\
\sum V_{i} T_{i}
\end{array}\right]
$$

The Type A uncertainty for $V_{\mathrm{z}}$ is computed as the standard deviation of the mean for the set

$$
V_{\mathrm{i}}-P_{\mathrm{i}} V_{\mathrm{z}}-V_{0}-m T_{\mathrm{i}} \quad \mathrm{i}=1 \text { to } 40 .
$$

Type B uncertainty includes components from the reference frequency, the null meter gain, leakage currents, and uncompensated offset voltages and is computed according to previously published guidelines [3].

The value of $V_{z}$ computed above contains information about the unknown reference voltage for only the relatively short time required to obtain the 40 data points (typically 5 minutes). The single measurement uncertainty is therefore not very relevant to the long term behavior of the unknown and serves more as an indicator of system performance. A reliable measurement of a Zener reference requires the analysis of many measurements made over a period of a week or more.

An internal simulator has been used to test the response of the calibration algorithm to a variety of nonideal behavior including thermal offsets and drifts, null meter gain, offset, and drift errors, step transitions, Gaussian noise, $1 / \mathrm{f}$ noise, outlying null measurements, etc.

There are manual bias controls for diagnostic purposes but in the normal operating mode the manual bias controls are disabled and there are no user adjustable controls. The system software includes control of all system parameters as well as complete self-calibration and self-diagnosis. For example, to calibrate the microwave subsystem, a microwave power meter is substituted for the array. The system then automatically measures the $77 \mathrm{GHz}$ power while driving the attenuator through its full range. A table of power vs. attenuator position is automatically recorded and used in all subsequent power adjustments. A self test function in the main menu provides a quantitative measurement of important system parameters including environmental conditions, bias system calibration, thermal offsets, and array critical current, leakage , and stability. Parameters that fall outside the acceptable range are flagged. Over 50 pages of help documentation are available on-line and can be easily searched for any word or phrase.

A prototype system has been constructed and will be shipped between NIST in Boulder, Colorado and Sandia National Laboratories in Albuquerque, New Mexico where its capabilities will be further evaluated against $10-\mathrm{V}$ laboratory Josephson array standards. It will then be shipped to selected NASA and DOE laboratories for field testing. The final version of the NASA and the DOE systems will be delivered in 1997.

\section{Acknowledgement}

The authors acknowledge many helpful suggestions from Richard Kautz and the loan of the Josephson array chips used in this system from Hypres Inc. 


\section{Figure Captions}

Figure 1. The physical layout of the Josephson voltage standard.

Figure 2. A block diagram of the portable JVS.

Figure 3. (a) A block diagram of the $77 \mathrm{GHz}$ phased locked oscillator and (b) a comparison of its line width (narrow spike) with the line width of a typical counter stabilized Gunn oscillator (broad peak).

Figure 4. A cross-sectional drawing showing the construction of the rigid dielectric waveguide used to transmit $77 \mathrm{GHz}$ power into the liquid helium Dewar.

Figure 5. The circuit used to compare the Josephson array and an unknown dc standard.

\section{$\underline{\text { References }}$}

[1] C. A. Hamilton, C.J. Burroughs, and K. Chieh, "Operation of NIST Josephson Array Voltage Standards," NIST Journal of Research, Vol. 95, pp. 219-235, April 1989.

[2] C. A. Hamilton, C.J. Burroughs, and K. Gilbert "NISTVolt - A Program for Automating Josephson Voltage Standards," available on request from NIST.

[3] "Josephson Voltage Standard," Recommended Intrinsic/Derived Standards Practice, RISP-1, April 1995, available from the National Conference of Standards Laboratories, Boulder, CO.

\section{DISCLAIMER}

This report was prepared as an account of work sponsored by an agency of the United States Government. Neither the United States Government nor any agency thereof, nor any of their employees, makes any warranty, express or implied, or assumes any legal liability or responsibility for the accuracy, completeness, or usefulness of any information, apparatus, product, or process disclosed, or represents that its use would not infringe privately owned rights. Reference herein to any specific commercial product, process, or service by trade name, trademark, manufacturer, or otherwise does not necessarily constitute or imply its endorsement, recommendation, or favoring by the United States Government or any agency thereof. The views and opinions of authors expressed herein do not necessarily state or reflect those of the United States Government or any agency thereof. 


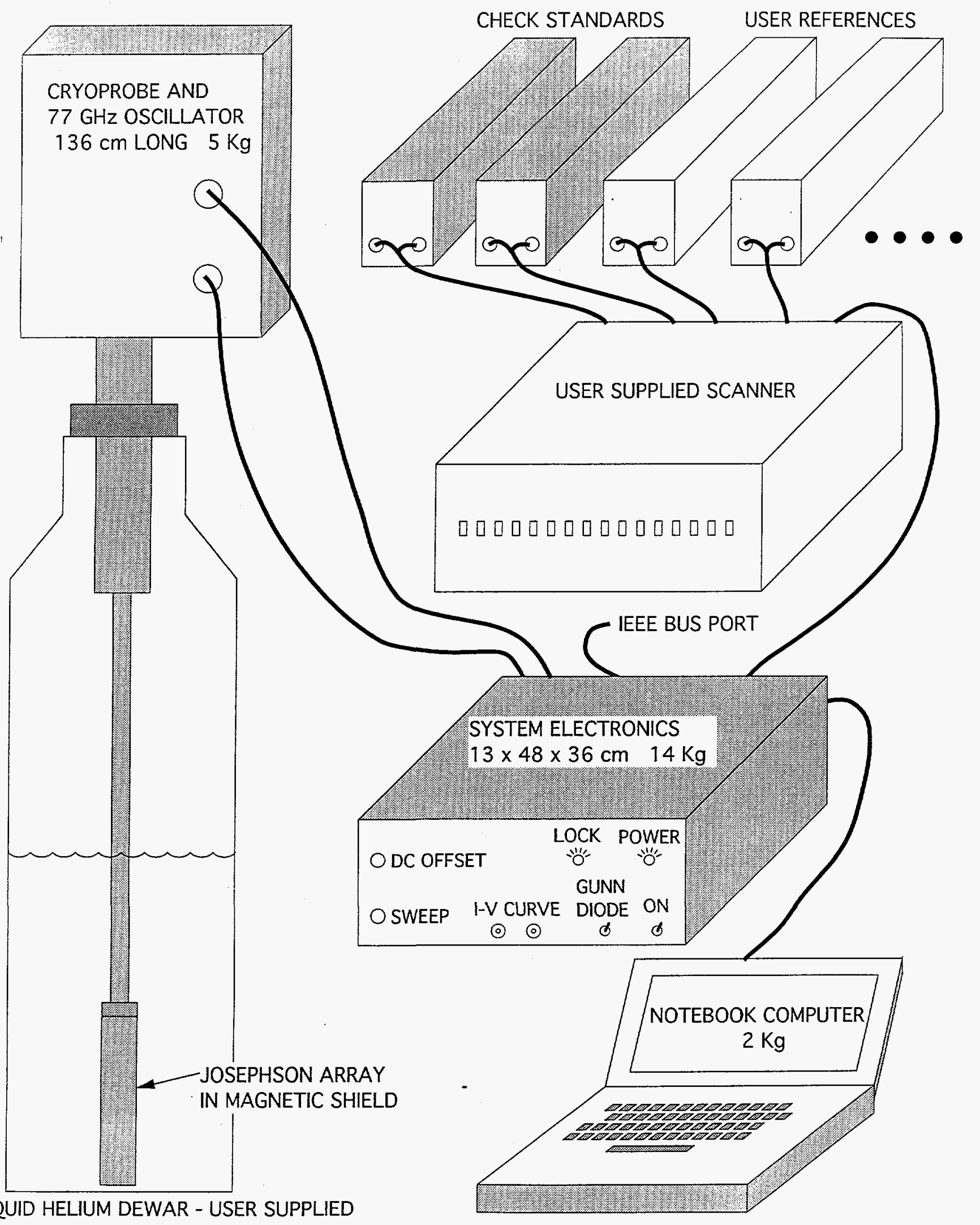


PORTABLE JOSEPHSON VOLTAGE STANDARD

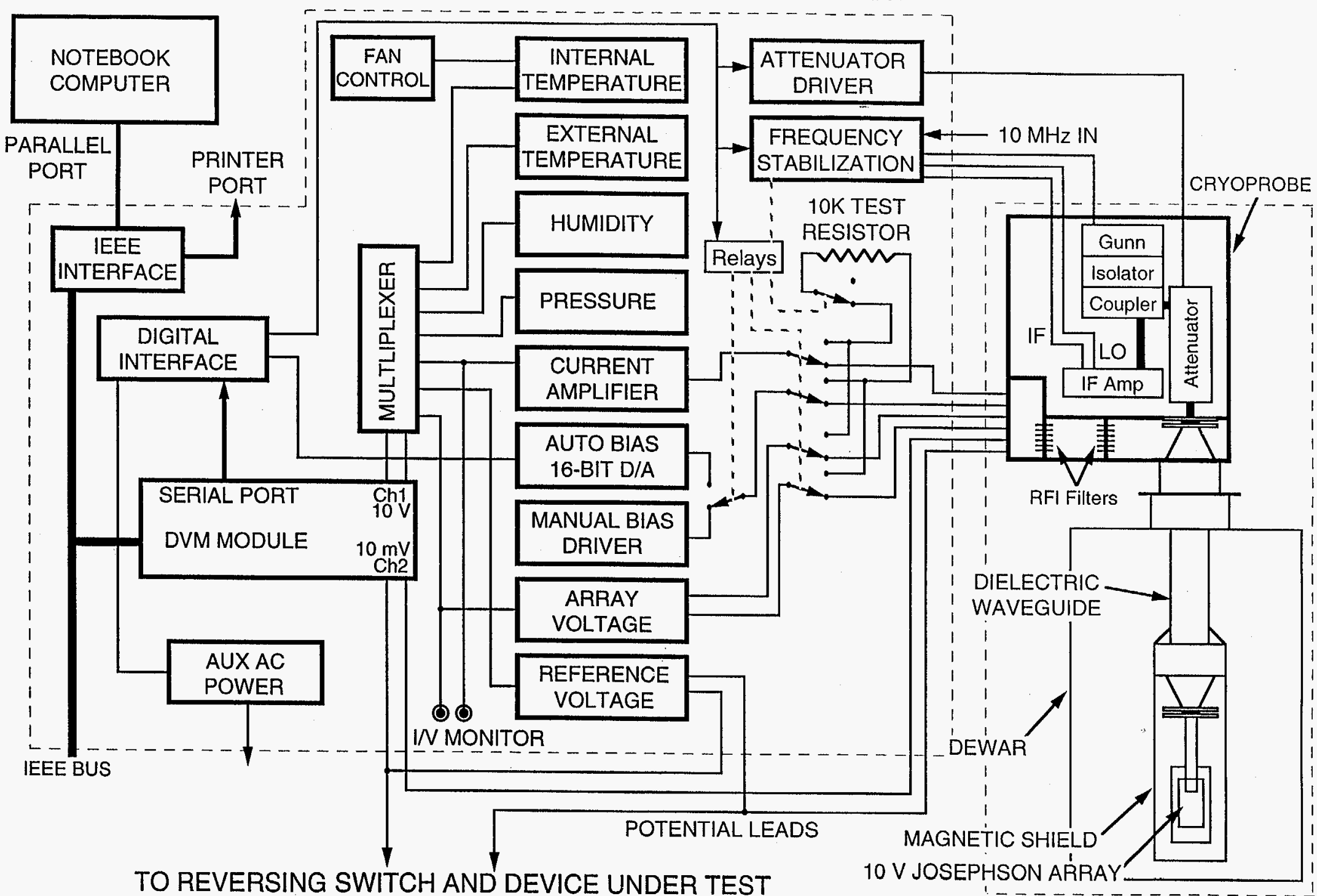



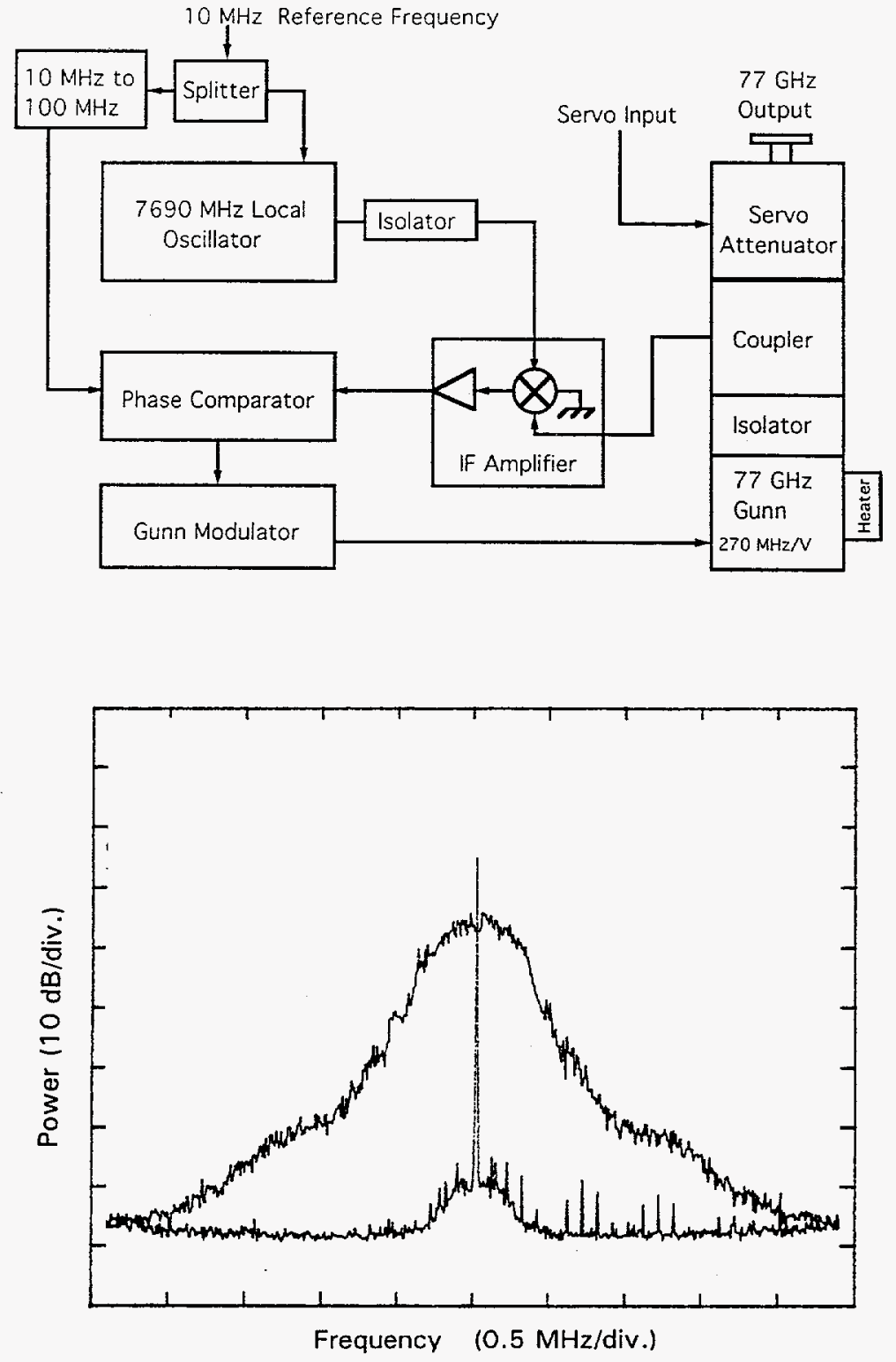


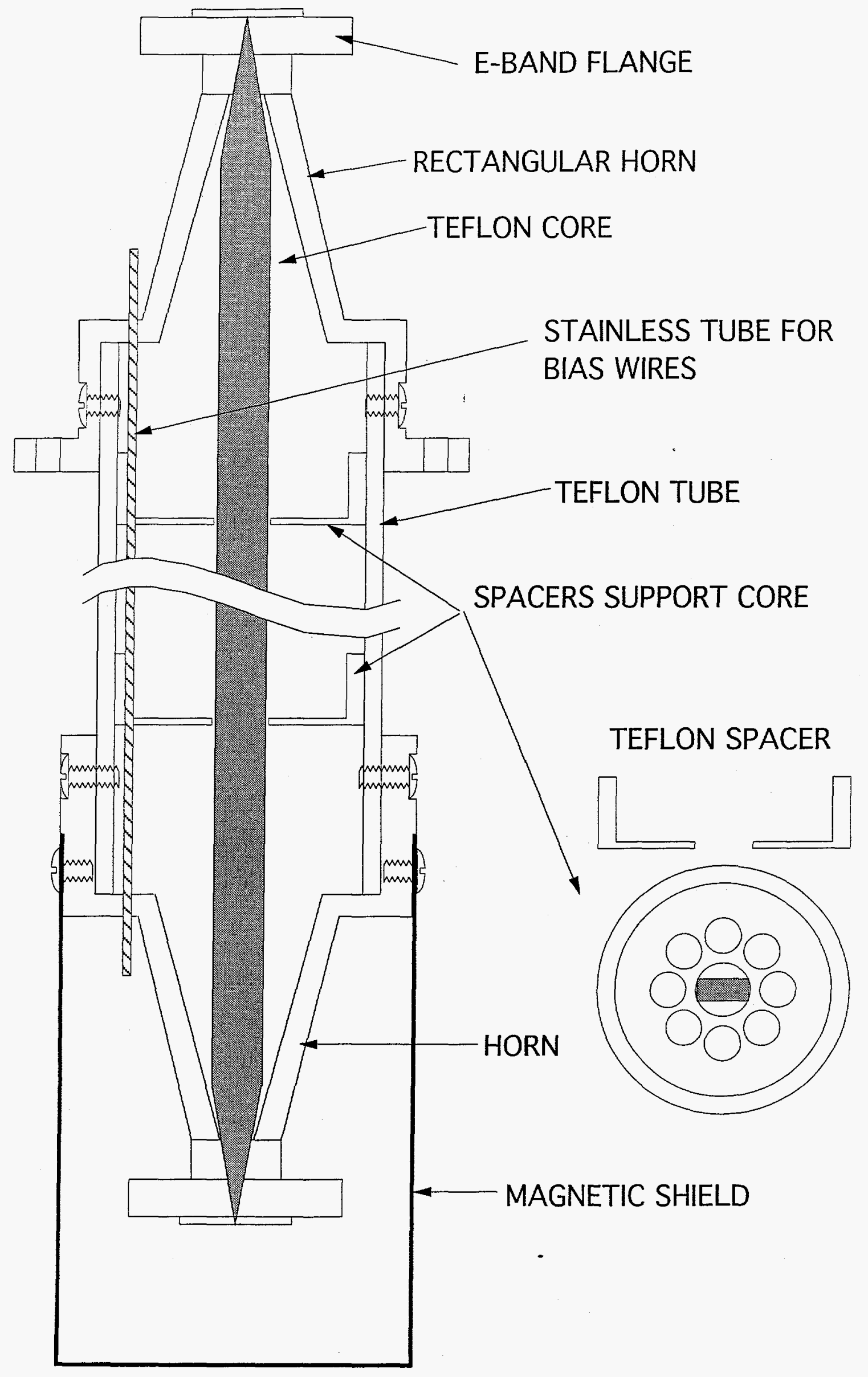




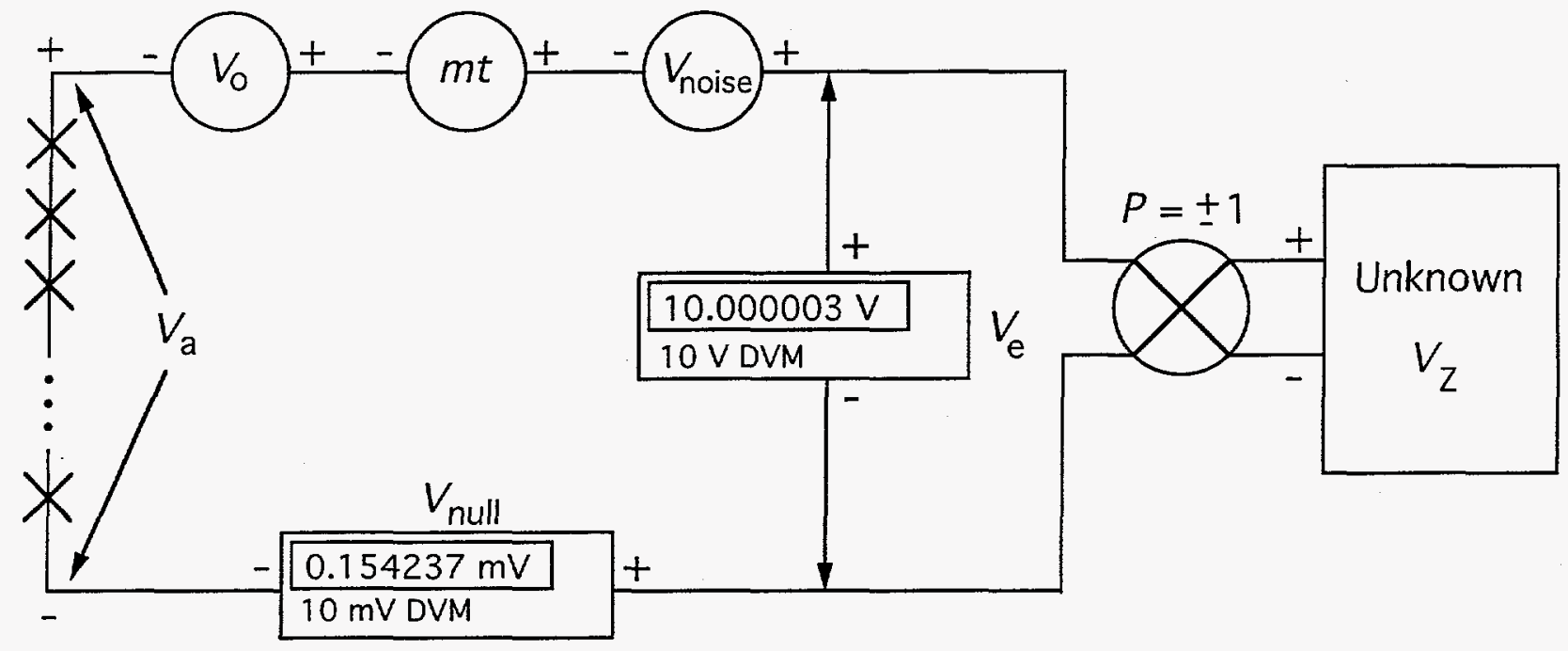

